\section{Acetylsalicylsäure stoppt Metastasierung}

Die tägliche Einnahme kleiner Dosen Acetylsalicylsäure (ASS) kann offenbar das Risiko senken, an Krebs zu sterben. Allerdings sind die Effekte eher kurzfristig. Eine mögliche Erklärung: ASS stoppt die Bildung von Fernmetastasen.

E rst vor kurzem hatte der Brite Peter E Rothwell untersucht, ob Patienten, die in mehrere randomisierte Studien zu vaskulären Ereignissen eingeschlossen waren, seltener an Krebs erkrankten, wenn sie sich im Aspirin- und nicht im Placebo-Arm befanden. Tatschlich reduzierte die tägliche Einnahme des Schmerz- und Entzündungshemmers die Wahrscheinlichkeit, in den folgenden fünf Jahren an Krebs zu sterben, um etwa $40 \%$. Nach 20 Jahren Follow-up war die Zahl krebsbedingter Todesfälle unter Aspirin signifikant vermindert.

Wenn der Langzeiteffekt so deutlich war, sollte dann nicht auch in kürzerer Zeit etwas zu sehen sein - etwa wenn die Patienten während der Studie an Krebs erkrankten? Rothwell war aufgefallen, dass der Effekt von ASS auf die Todesrate größer war als der Effekt auf die Krebsinzidenz. Zudem war die geringere Mortalität zu schnell zu erkennen (schon nach fünf Jahren, beim kolorektalen Karinom [CRC] noch schneller), als dass sie sich ausschließlich durch einen Effekt auf Karzinogenese oder frühes Tumorwachstum erklären ließe. Drittens schien auch die Mortalität durch metastasierte Tumoren reduziert, ohne dass sich dies zunächst erklären ließ.

Deshalb analysierte Rothwell die Ergebnisse aller fünf großen, britischen, randomisierten Studien, in denen tägliches Aspirin ( $\geq 75 \mathrm{mg} / \mathrm{Tag}$ ) gegenüber Placebo für die Prävention von vaskulären Ereignissen untersucht wurde.

Bei 987 der 17.285 Probanden wurde während des medianen Follow-ups von 6,5 Jahren ein solider Tumor diagnostiziert. Die Teilnahme in der Aspiringruppe reduzierte das Risiko für einen Krebs mit Fernmetastasen (Hazard Ratio [HR] 0,64 ); wurden nur Adenokarzinome betrachtet, lag die HR bei 0,54; für andere solide Tumoren bei 0,82 .
Lagen bei der initialen Diagnose noch keine Metastasen vor, betrug die HR für eine Metastasierung 0,45, für Patienten mit CRC sogar nur 0,26. Das Todesrisiko der Patienten, die ein Adenokarzinom entwickelten, sank unter Aspirin signifikant $(H R=0,50)$. Das Gesamtrisiko für ein tödliches Adenokarzinom lag damit in der Aspirin-Gruppe bei 0,65. ASS senkte aber nicht das Risiko für andere tödliche Krebserkrankungen $(\mathrm{HR}=$ 1,06). Der absolute Benefit war für Raucher am größten.

Fazit: Dass tägliches ASS das Risiko senkt, an Krebs zu sterben, ließ sich bislang kaum praktisch nutzen, wollte man nicht die gesamte Bevölkerung unter Medikation stellen. Bestätigt sich, dass Aspirin die Metastasierung (vor allem von Adenokarzinomen) hemmt, ergibt sich doch eine therapeutische Option. In jedem Fall sollte das Absetzen von Aspirin nach einer Krebsdiagnose sehr kritisch beleuchtet werden. Christina Berndt

Rothwell PM et al. Effect of daily aspirin on risk of cancer metastasis: A study of incident cancers during randomised controlled trials. Lancet. 2012;379(9826):1591-601.

\title{
Infektionen verursachen jeden sechsten Krebs
}

\section{Rund zwei der weltweit 12,7 Millionen Krebserkrankungen pro Jahr sind durch Infektionenn verursacht, haben Forscher der International Agency for Research on Cancer (IARC) der Weltgesundheitsorganisation WHO errechnet.}

D ie Wissenschaftler griffen für ihre Auswertung auf die Daten des GLOBOCAN-Projektes aus dem Jahr 2008 in acht Regionen zurück: Nordafrika/westliches Asien, Afrika südlich der Sahara, Zentral- und Ostasien, Nord- und Südamerika, Europa sowie Ozeanien. Insgesamt ergab sich, dass die für 2008 infektiösen Auslösern zuzuordnende Rate an Krebserkrankungen bei 16,1\% liegt das entspricht zwei Millionen der insgesamt 12,7 Millionen Fälle. Für weniger entwickelte Länder betrug die Rate $22,9 \%$, in entwickelten Regionen lag sie bei $7,4 \%$.

Der größte Anteil - 1,9 Millionen Fälle - ging auf Infektionen mit Helicobacter (H.) pylori, Hepatitis-B- und -C-Viren (HBV, HCV) sowie humane Papil- lomviren (HPV) zurück, betroffen waren vor allem Magen, Leber und Zervix. Rund die Hälfte der infektbedingten Krebstumoren von Frauen entfiel auf Zervixkarzinome; bei den Männern $80 \%$ auf Magen- und Leberkrebs. 30 von 100 Betroffenen waren jünger als 50 Jahre. $\mathrm{Zu}$ den bevorzugten Lokalisationen zählten —Magen: H. pylori

_Leber: HBV, HCV, die Leberegel Opisthorchis viverrini und Clonorchis sinensis

_Cervix uteri: HPV mit oder ohne HIV _ Anogenitale: HPV mit oder ohne HIV -Nasopharynx: Epstein-Barr-Virus (EBV)

-Oropharynx: HPV mit oder ohne Tabak- bzw. Alkoholkonsum

_Haut/Schleimhäute (Kaposi-Sarkom): humanes Herpesvirus Typ 8 mit oder ohne HIV

_blutbildendes System (Non-HodgkinLymphom): H. pylori, EBV mit oder ohne HIV, HCV, HTLV-1

_blutbildendes System (Hodgkin-Lymphom): EBV mit oder ohne HIV

-Blase: der Saugwurm Schistosoma haematobium

Fazit: Gut $16 \%$ aller Tumoren gehen auf Infektionen zurück, der Kampf gegen Infektionen, v.a. H. pylori, HBV, HCV und HPV, ist damit auch ein Kampf gegen den Krebs. Die Kollegen vom IARC plädieren für Prävention durch Impfungen, mehr Sicherheit bei Injektionen und antimikrobielle Therapie. Diese Maßnahmen könnten sich wesentlich auf die künftige Entwicklung der weltweiten Krebszahlen auswirken. Robert Bublak

De Martel C et al. Global burden of cancers attributable to infections in 2008: A review and synthetic analysis. Lancet Oncol. 2012;13(6):607-15. 